\title{
Solitary waves on nonlinear elastic rods. II.
}

\section{Sørensen, Mads Peter; Christiansen, Peter Leth; Lomdahl, P. S.; Skovgaard, Ove}

\section{Published in:}

Acoustical Society of America. Journal

Link to article, DOI:

10.1121/1.394786

Publication date:

1987

\section{Document Version}

Publisher's PDF, also known as Version of record

Link back to DTU Orbit

Citation (APA):

Sørensen, M. P., Christiansen, P. L., Lomdahl, P. S., \& Skovgaard, O. (1987). Solitary waves on nonlinear elastic rods. II. Acoustical Society of America. Journal, 81(6), 1718-1722. https://doi.org/10.1121/1.394786

\section{General rights}

Copyright and moral rights for the publications made accessible in the public portal are retained by the authors and/or other copyright owners and it is a condition of accessing publications that users recognise and abide by the legal requirements associated with these rights.

- Users may download and print one copy of any publication from the public portal for the purpose of private study or research.

- You may not further distribute the material or use it for any profit-making activity or commercial gain

- You may freely distribute the URL identifying the publication in the public portal

If you believe that this document breaches copyright please contact us providing details, and we will remove access to the work immediately and investigate your claim 


\author{
M. P. Soerensen,a) P. L. Christiansen, P. S. Lomdahl, ${ }^{\text {b) }}$ and O. Skovgaard \\ Laboratory of Applied Mathematical Physics, The Technical University of Denmark, DK-2800 Lyngby, \\ Denmark
}

(Received 22 January 1986; accepted for publication 14 January 1987)

\begin{abstract}
In continuation of an earlier study of propagation of solitary waves on nonlinear elastic rods, numerical investigations of blowup, reflection, and fission at continuous and discontinuous variation of the cross section for the rod and reflection at the end of the rod are presented. The results are compared with predictions of conservation theorems for energy and momentum.
\end{abstract}

PACS numbers: 43.25.Dc, 43.25.Jh, 43.40.Cw

\section{INTRODUCTION}

In a preceding paper, ${ }^{1}$ which we shall denote as Paper I in the following sections, we investigated the propagation of nonlinear acoustical waves on a circular rod. The radial displacement and the nonlinearity of the material are taken into account by including a fourth-order derivative of the displacement and by including terms up to fourth order in the Taylor expansion of the elastic energy, respectively. As a result we obtain the so-called improved Boussinesq equation, denoted by IBE, in the case of a quadratic nonlinearity and the modified Boussinesq equation, denoted by MIBE, in the case of a cubic nonlinearity, respectively.

Solutions to Korteweg-de Vries equations with varying coefficients have been studied by a number of authors by perturbative and numerical methods. ${ }^{2-6}$ Studies of equations related to IBE and MIBE are found in Ref. 7 considering the inhomogeneous rod with varying cross section, density, and Young's modulus, Refs. 8 and 9, in which both quadratic and cubic nonlinearities are considered in a generalized Boussinesq equation (GBE), and Ref. 10 devoted to a version of the improved Boussinesq equation, which contains the nonlinearity in the fourth-order term.

In the present article we first investigate blowup of different negative solitary wave solutions to IBE. In Ref. 11 the nonexistence of global positive solutions to the Dirichlet problem for the Boussinesq equation $u_{t t}=3 u_{x x x x}+u_{x x}$ $-12\left(u^{2}\right)_{x x}$ is proved. This article contains a number of references to works on existing proofs. The effect of replacing $\partial_{x}$ by $\partial_{t}$ on the existence of solutions has been studied in Ref. 12.

The following sections contain studies of reflection as well as fission of solitary waves in the MIBE case at continuously varying cross sections of the elastic rod. In the latter case the relevant nonlinear interface boundary conditions are derived by an argument from calculus of variations.

In the final section we investigate reflection of solitary waves at the end of the elastic rod in two cases corresponding to almost free- and fixed-end boundary conditions. Our nu-

\footnotetext{
a) Present address: IBM Forschungslaboratorium Zürich, Säumerstrasse 4 , CH-8803, Rüschlikon, Switzerland.

b) Present address: Center for Nonlinear Studies, Los Alamos National Laboratory, University of California, Los Alamos, NM 87545.
}

merical results are compared with the predictions of conservation theorems for energy and momentum.

\section{WAVE EQUATIONS AND CONSERVED QUANTITIES}

In Paper I we considered the longitudinal displacement component $W(X, T)$ of a plane cross section along the isotropic circular cylindrical rod. Here, $X$ is the position of the undisturbed cross section and $T$ is time. When the width of the solitary wave is large compared with the radius of the rod, we may assume that the radial displacement $U(X, R, T)$ in the time-dependent inhomogeneous case is given by the Taylor series

$$
U(X, R, T)=-\sigma_{1} W_{X}-\left(\sigma_{2} / 2 !\right) W_{X}^{2}-\cdots .
$$

Here, $R$ is the radial variable and the expansion coefficients, $\sigma_{1}, \ldots$, are the first- and higher-order Poisson ratios. The elastic energy density to the fourth order becomes

$$
\phi=\frac{1}{2 !} E_{2} W_{X}^{2}+\frac{1}{3 !} E_{3} W_{X}^{3}+\frac{1}{4 !} E_{4} W_{X}^{4},
$$

where $E_{2}$, Young's modulus, and the higher-order expansion coefficients, $E_{3}$ and $E_{4}$, are given in Paper I (Appendix A). The Lagrangian density for the system becomes

$$
\begin{aligned}
\mathscr{L}= & \frac{1}{2} S \rho\left[W_{T}^{2}-\left(S \sigma_{1}^{2} / 2 \pi\right) W_{X T}^{2}\right] \\
& -\frac{1}{2} S E_{2} W_{X}^{2}-\frac{1}{6} S E_{3} W_{X}^{3}-\frac{1}{24} S E_{4} W_{X}^{4},
\end{aligned}
$$

where $S$ is the cross-sectional area and $\rho$ is the density of the elastic material. The coefficients $E_{2}, E_{3}, E_{4}, \sigma_{1}, S$, and $\rho$ may depend on $X$. In Paper I the coefficients were constants. In the present paper $E_{2}, E_{3}, E_{4}$, and $\sigma_{1}$ are constants, while the cross-sectional area $S$ and the density $\rho$ vary. In the case of continuous variation of $S$ and $\rho$ the Euler equation for this Lagrangian density is

$$
\frac{\partial}{\partial X} \frac{\partial \mathscr{L}}{\partial W_{X}}+\frac{\partial}{\partial T} \frac{\partial \mathscr{L}}{\partial W_{T}}-\frac{\partial^{2}}{\partial X \partial T} \frac{\partial \mathscr{L}}{\partial W_{X T}}=0,
$$

yielding

$$
\begin{gathered}
E_{2} \frac{\partial}{\partial X}\left(S W_{X}\right)+\frac{1}{2} E_{3} \frac{\partial}{\partial X}\left(S W_{X}^{2}\right)+\frac{1}{3} E_{4} \frac{\partial}{\partial X}\left(S W_{X}^{3}\right) \\
-\rho \frac{\partial}{\partial T}\left(S W_{T}\right)+\frac{\sigma^{2}}{2 \pi} \frac{\partial^{2}}{\partial X \partial T}\left(\rho S^{2} W_{X T}\right)=0 .
\end{gathered}
$$

In the case of discontinuous variation of $S$ and $\rho$ such that $S=S_{1}$ and $\rho=\rho_{1}$ for $X<X_{0}$, and $S=S_{2}$ and $\rho=\rho_{2}$ for $X>X_{0}$, Eq. (5) is replaced by 


$$
\begin{gathered}
E_{2} \frac{\partial}{\partial X} W_{X}+\frac{1}{2} E_{3} \frac{\partial}{\partial X}\left(W_{X}^{2}\right)+\frac{1}{3} E_{4} \frac{\partial}{\partial X}\left(W_{X}^{3}\right) \\
\quad-\rho_{i} \frac{\partial}{\partial T}\left(W_{T}\right)+\frac{\rho_{1} \sigma^{2} S_{i}}{2 \pi} \frac{\partial^{2}}{\partial X \partial T} W_{X T}=0, \\
i=\left\{\begin{array}{l}
1 \\
2
\end{array} \text { or } X \lessgtr X_{0}\right. \text { and } \\
\left(E_{2} S_{i} W_{X}+\frac{1}{2} E_{3} S_{i} W_{X}^{2}+\frac{1}{3} E_{4} S_{i} W_{X}^{3}\right. \\
\left.\quad+\frac{\rho_{i} \sigma^{2} S_{i}^{2}}{2} W_{X T T}\right)_{X_{0}-}^{X_{0}+}-\int_{X_{0}-}^{X_{0}+} \rho_{i} S_{i} W_{T T} d X=0 .
\end{gathered}
$$

This result follows from Hamilton's principle ${ }^{13}$ for the elastic rod. In the present paper we shall solve Eq. (5) in the following particular cases. to IBE,

(i) $E_{4}=0, E_{2}>0, E_{3}>0, S$ and $\rho$ are constants leading

$$
u_{x x}-u_{x t}+\frac{1}{2}\left(u^{2}\right)_{x x}+u_{x x t}=0
$$

for the dimensionless strain $u=w_{x}$, where

$w(x, t)=W(X, T) E_{3} /\left(E_{2} \sigma \sqrt{S / 2 \pi}\right), \quad x=X /(\sigma \sqrt{S / 2 \pi})$, and

$$
t=T \sqrt{E_{2}} /(\sigma \sqrt{S \rho / 2 \pi}) .
$$

(ii) $E_{3}=0, E_{2}>0$, and $E_{4}>0$ are constants, and $S=s(x) S_{1}$, where $S_{1}$ is a reference cross section and $s(x)$ is a dimensionless continuously varying cross-sectional function leading to

$$
\left(s w_{x}\right)_{x}+\frac{1}{3}\left(s w_{x}^{3}\right)_{x}-s w_{t c}+\left(s^{2} w_{x t}\right)_{x t}=0,
$$

where

$$
\begin{aligned}
& w(x, t)=W(X, T) \sqrt{E_{4}} /\left(\sigma \sqrt{S_{1} E_{2} / 2 \pi}\right), \\
& x=X /\left(\sigma \sqrt{S_{1} / 2 \pi}\right),
\end{aligned}
$$

and

$$
t=T \sqrt{E_{2}} /\left(\sigma \sqrt{S_{1} \rho / 2 \pi}\right) .
$$

In the discontinuous case, Eq. (6) is solved in the following cases. while

(iii) $E_{3}=0, E_{2}>0, E_{4}>0$, and $\rho>0$ are constants,

$$
S=\left\{\begin{array}{l}
S_{1} \\
S_{2}
\end{array} \text { for } x \lessgtr x_{0}\right.
$$

leading to

$w_{x x}-w_{t t}+\frac{1}{3}\left(w_{x}^{3}\right)_{x t}+\left[\begin{array}{l}1 \\ S_{2} / S_{1}\end{array}\right] w_{x x t t}=0, \quad$ for $x \lessgtr x_{0}$,

with the dimensionless variables $w, x$, and $t$ given in the same manner as in case (ii), and the transition condition

$$
\left[\frac{S_{i}}{S_{1}}\left(w_{x}+\frac{1}{3} w_{x}^{3} \frac{S_{i}}{S} w_{x t}\right)\right]_{x_{0}-}^{x_{0}+}=0
$$

with

$$
i=\left\{\begin{array}{l}
1 \\
2
\end{array} \text { for } x \lessgtr x_{0}=X_{0} /\left(\sigma \sqrt{S_{1} / 2 \pi}\right) .\right.
$$

(iv) $E_{3}=0, E_{2}>0$, and $E_{4}>0$ are constants, while
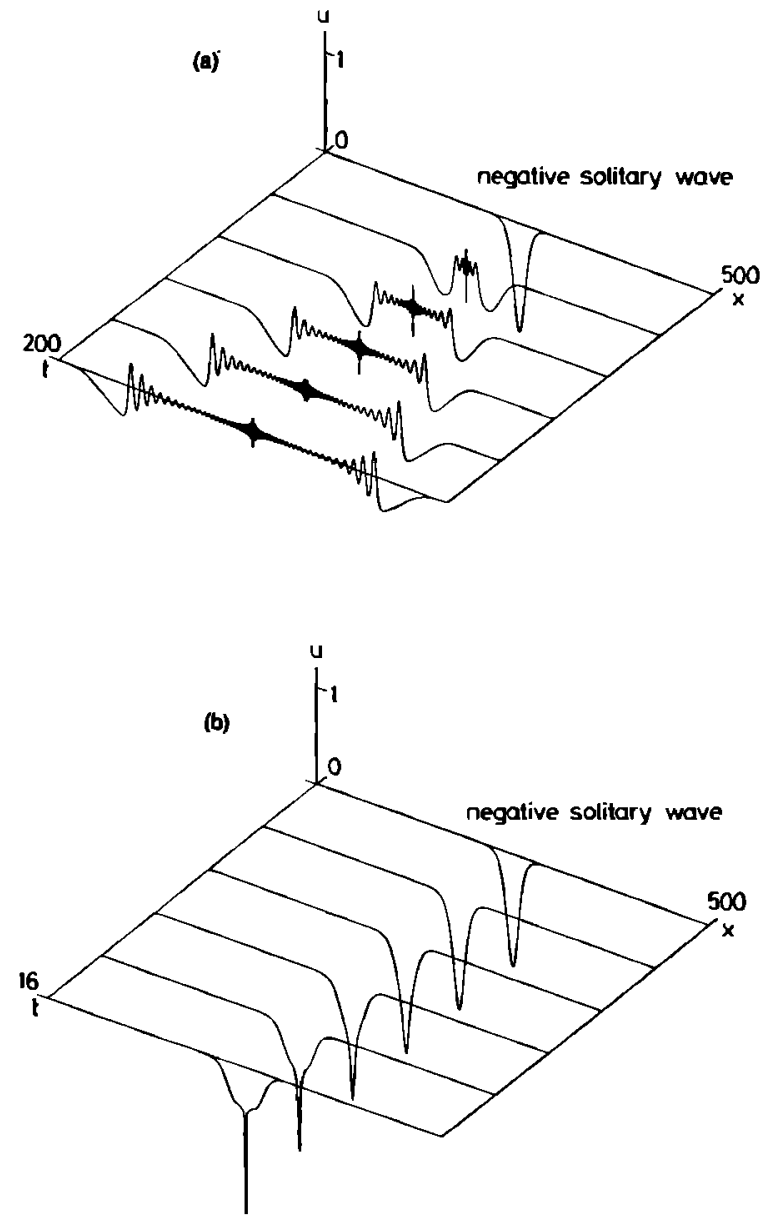

FIG. 1. Time evolution of negative static solitary wave for IBE. Numerical solutions of (7) with initial condition (15). (a) Dispersive case, $A=-1.1, B=0.1$. (b) Blowup case, $A=-1.11, B=0.1$.

$$
\begin{aligned}
& S=\left\{\begin{array}{l}
S_{1} \\
0
\end{array}\right. \text { and } \\
& \rho=\left\{\begin{array}{l}
\rho_{1} \\
\rho_{1}+\left(M / S_{1}\right) \delta\left(x-x_{0}\right)
\end{array} \quad \text { for } x \lessgtr x_{0},\right.
\end{aligned}
$$

where $\delta\left(x-x_{0}\right)$ denotes Dirac's delta function. This corresponds to a homogeneous rod ending at $x=x_{0}$ and loaded by the mass $M$ placed at the point $x=x_{0}$. The mathematical formulation becomes

$$
w_{x x}-w_{u t}+\frac{1}{3}\left(w_{x}^{3}\right)_{x}+w_{x x u}=0
$$

with the dimensionless variables $w, x$, and $t$ given as in case

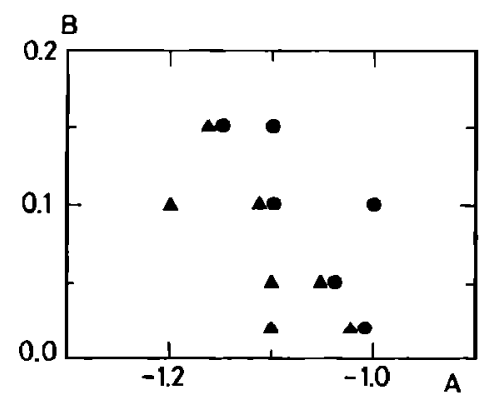

FIG. 2. Dependence of numerical solutions of IBE (7) on parameters $A$ and $B$ in initial condition (15). Blowup: $A$. Dispersion: $\bullet$. 


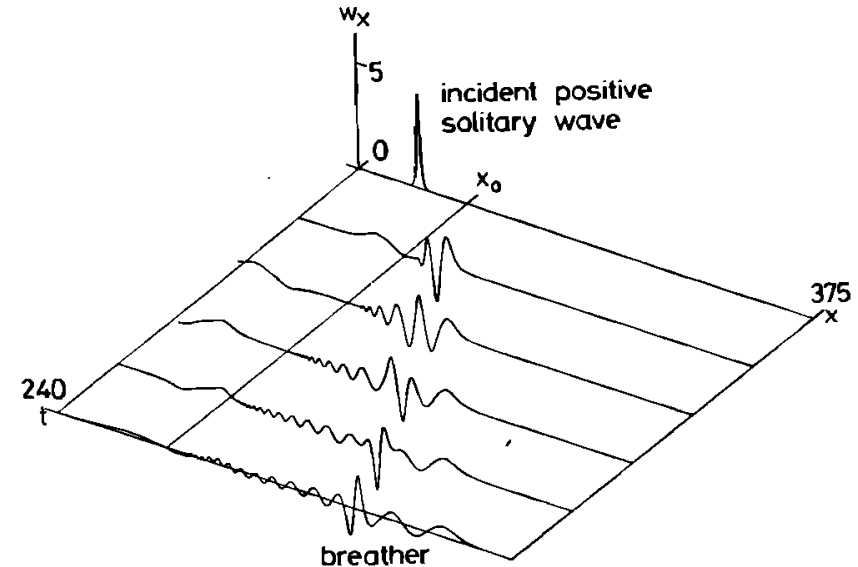

FIG. 3. Destruction of solitary wave at continuously increasing cross section. Numerical solutions of (8) and (16), and initial condition (17). $S_{2} / S_{1}=5, \alpha=0.1, c=2$, displayed in terms of $w_{x}$.

(ii) with $\rho$ replaced by $\rho_{1}$ in the formula for $t$ and the boundary condition

$$
w_{x}+\frac{1}{3} w_{x}^{3}-w_{x t t}-m w_{t t}=0, \text { for } x=x_{0},
$$

with the dimensionless mass $m=M /\left(\sigma S_{1}^{3 / 2} \rho_{1} / \sqrt{2 \pi}\right)$.

In Paper I expressions for total energy $H$, total momentum $P$, and total displacement $D$ are derived, and it is shown that $H, P$, and $D$ are conserved at IBE and MIBE. Here, we insert the solitary wave solution

$$
w(x, t)= \pm \sqrt{6} c \tan ^{-1}\left(\sinh \frac{\sqrt{c^{2}-1}}{c}\left(x-x_{p}-c t\right)\right),
$$

where $c$ is the velocity of the wave, $x_{p}$ is the position at $t=0$, and $w_{x}$ satisfies MIBE, into these expressions and obtain

$$
\begin{aligned}
& H=\left(E_{2}^{2} \sigma S_{1} \sqrt{S_{1} / 2 \pi} / E_{4}\right) h, \\
& h=12 c^{2}|c|\left(c^{2}-1\right)^{1 / 2} s^{3 / 2}, \\
& P=\left(E_{2} \sigma S_{1} \sqrt{p S_{1} / 2 \pi} / \sqrt{E_{4}}\right) p,
\end{aligned}
$$

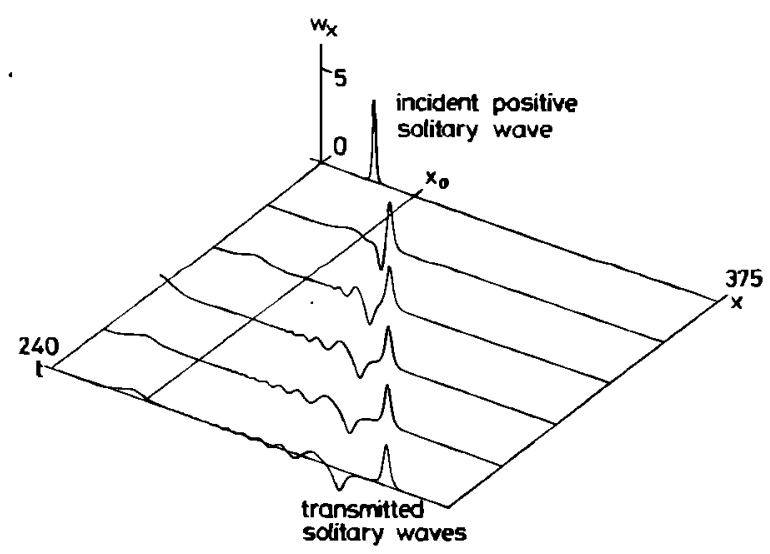

FIG. 4. Fission into solitary and antisolitary waves at continuously increasing cross section. Numerical solutions of $(8)$ and (16), initial condition (17). $S_{2} / S_{1}=3, \alpha=0.1, c=2$, displayed in terms of $w_{x}$.

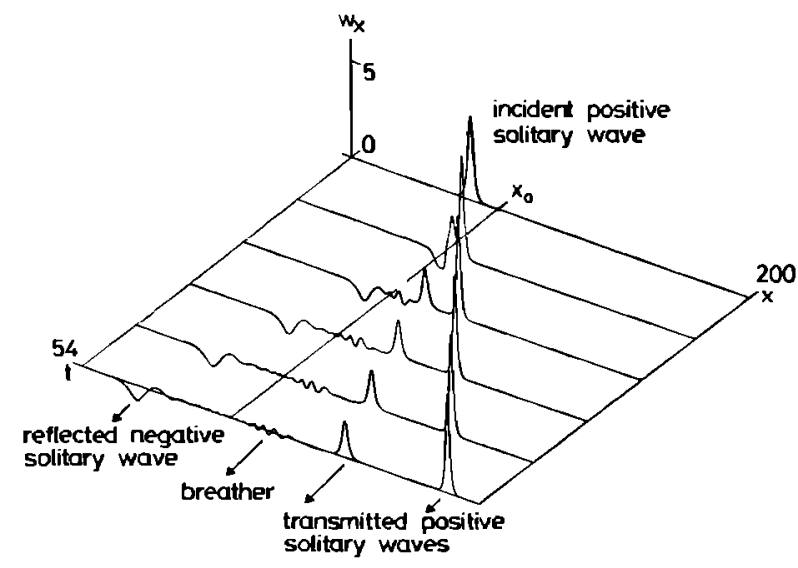

FIG. 5. Fission into two solitary waves and reflection of antisolitary wave at continuously decreasing cross section. Numerical solutions of (8) and (16), initial condition (17). $S_{2} / S_{1}=0.6, \alpha=1, c=2$, displayed in terms of $w_{x}$.

$$
\begin{aligned}
& p=\mp \pi \sqrt{6}|c| c s^{3 / 2}, \\
& D=\left(\sigma \sqrt{S_{1} E_{2} / 2 \pi} / \sqrt{E_{4}}\right) d, \\
& d= \pm \pi \sqrt{6}|c| s^{1 / 2},
\end{aligned}
$$

with upper and lower signs for solitary wave and antisolitary wave, respectively, and $|c| \geqslant 1$.

\section{BLOWUP OF SOLUTIONS AT IBE}

In the case of a quadratic nonlinearity IBE (7) may lead to dispersive or blowup destruction of negative static initial pulses

$$
\left.\begin{array}{l}
u(x, 0)=A \operatorname{sech} B\left(x-x_{0}\right) \\
u_{t}(x, 0)=0
\end{array}\right\},
$$

as shown in Fig. 1 (a) and (b), respectively. The dependence of the numerical solution of IBE (7) on parameters $A$ and $B$ in initial condition (15) is illustrated in Fig. 2. Here, blowup

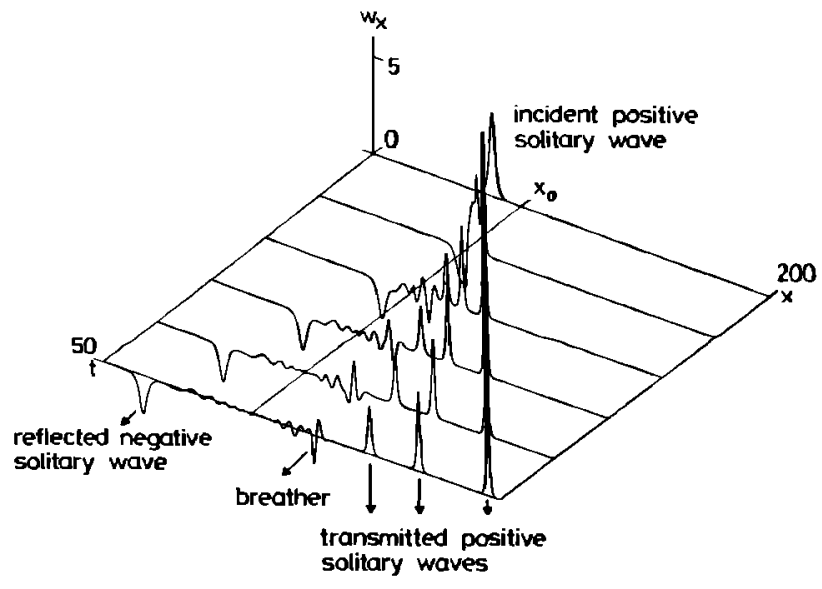

FIG. 6. Fission into three solitary waves, breather, and reflection of antisolitary wave at continuously decreasing cross section. Numerical solutions of (8) and (16), initial condition (17). Here, $S_{2} / S_{1}=0.3, \alpha=1, c=2$, displayed in terms of $w_{x}$. 


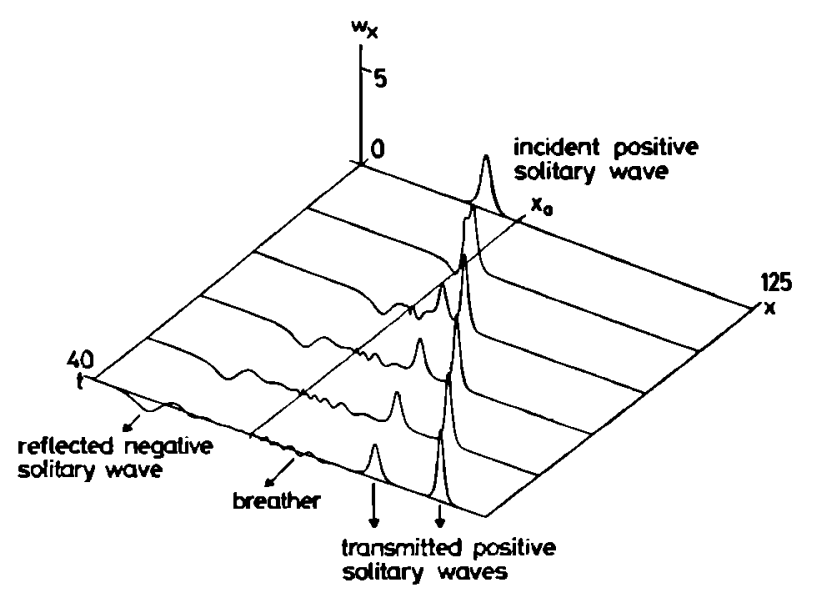

FIG. 7. Fission into two solitary waves and reflection of antisolitary wave at discontinuously decreasing cross section. Numerical solution of (9), initial condition (17). Here, $S_{2} / S_{1}=0.49, c=1.5$, displayed in terms of $w_{x}$.

and dispersion of the solution are indicated by $\mathbf{\Delta}$ and $\boldsymbol{O}$, respectively. The area of the initial pulse is proportional to $A / B$, while the curvature at the extremum point is proportional to $A B^{2}$. In $A B$-parameter space (Fig. 2) the boundary between the blowup region and the dispersion region appears to be a tilted straight line. This indicates that the area of the initial pulse (rather than the extremum or the the extrema curvature of the pulse) decides whether blowup or dispersion occurs or not. An analytical confirmation of this observation requires a detailed examination of the existence of solution to IBE.

At IBE, transmission and reflection of solitary waves do not lead to antisolitary waves since such waves are not solutions to IBE. However, the possibility of blowup of solutions in this case makes such a study difficult from a numerical point of view. In the remaining part of this article we therefore restrict the investigation of scattering phenomena to the MIBE case.

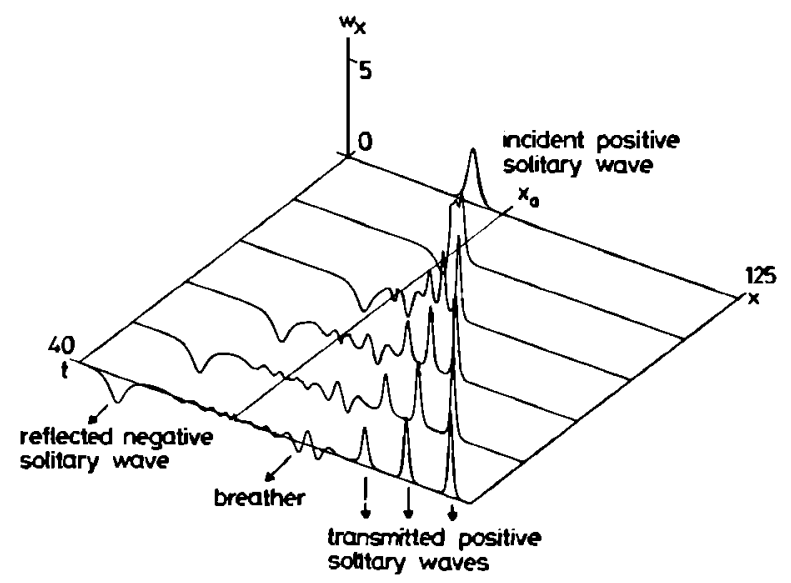

FIG. 8. Fission into three solitary waves, breather, and reflection into antisolitary wave at discontinuously decreasing cross section. Numerical solution of (9), initial condition (17). Here, $S_{1} / S_{2}=0.25, c=1.5$, displayed in terms of $w_{x}$.

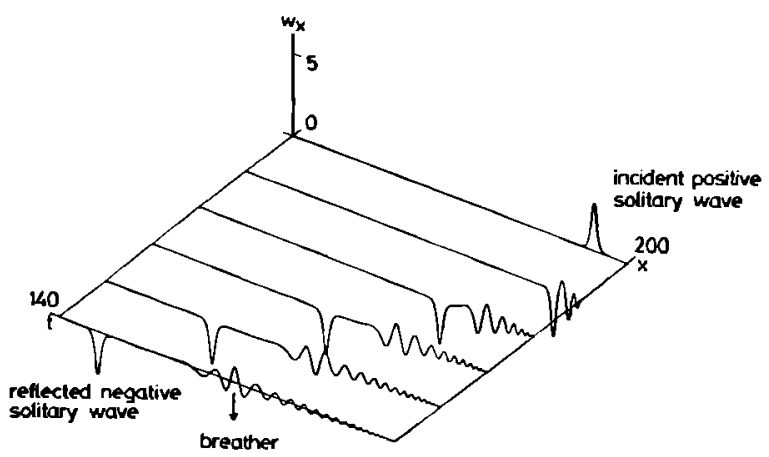

FIG. 9. Reflection of solitary wave at weakly loaded end. Numerical solution of (10), initial condition (17). Here, $m=5, c=1.5$, displayed in terms of $w_{\mathbf{x}}$.

\section{CONTINUOUSLY VARYING CROSS SECTION AT MIBE}

In the case of a cubic nonlinearity (8) and continuously varying cross section

$$
s(x)=1+\left[\left(S_{2}-S_{1}\right) / 2 S_{1}\right]\left\{\tanh \left[\alpha\left(x-x_{0}\right)\right]+1\right\},
$$

we investigate the propagation of a solitary wave given by $w(x, t)=\sqrt{6} c \tan ^{-1}\left\{\sinh \left[\left(\sqrt{c^{2}-1} / c\right)\left(x-x_{p}-c t\right)\right]\right\}$,

which is a solution to (8) for $s=1$.

The dimensionless cross-sectional function $s(x)$ varies from $s(-\infty)=1$ through $s\left(x_{0}\right)=\left(S_{1}+S_{2}\right) / 2$ to $s(\infty)=S_{2} / S_{1}$, the steepness being $d s / d x=\alpha\left(S_{2}-S_{1}\right) /$ $2 S_{1}$ at $x=x_{0}$. The position and the velocity of the incident solitary waves, (17), are given by $x=x_{p}$ at $t=0$ and $c$, respectively.

Figure 3 shows the destruction of the incident solitary wave $(c=2)$ at the point where the cross-sectional area is increased $\left(S_{2} / S_{1}=5, \alpha=0.1\right)$. For the incident wave the normalized momentum (13) is $p=-\pi \sqrt{6} \cdot 2^{2}=30.8$. According to the same formula a transmitted solitary wave would require at least $p=-\pi \sqrt{6} \cdot 1^{2} 5^{3 / 2}=-86.0$. This transmission is not possible in the present case.

In Fig. 4, parameters are $S_{2} / S_{1}=3, \alpha=0.1$, and $c=2$. Here, a transmitted solitary wave, a transmitted antisolitary

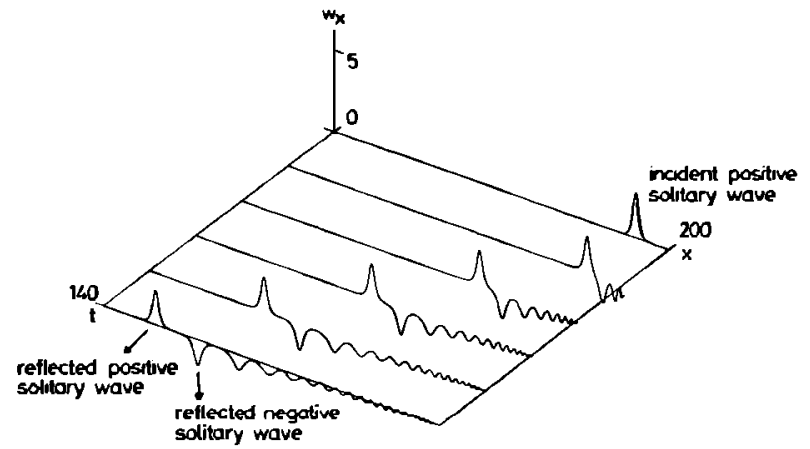

FIG. 10. Reflection of solitary wave at heavily loaded end. Numerical solution of (10), initial condition (17). Here, $m=25, c=1.5$, displayed in terms of $w_{x}$. 
wave, a breather, and a reflected wave are produced. The normalized momentum of the incident solitary wave is still $p_{\text {inc }}=-30.8$. Transmitted solitary and antisolitary waves possess total momentum of $-69.7+47.5=-22.2$. Thus momentum of 8.6 is available for the reflected wave (and the breather).

In Fig. 5 the cross section of the right half of the rod is reduced to $S_{2} / S_{1}=0.6(\alpha=1$ and $c=2)$. As a result the incident solitary wave is fissioned into two transmitted solitary waves, and an antisolitary wave is reflected from the cross-sectional change at $x=x_{0}$. Also, a breather is produced. Again, the normalized momentum of the incident solitary wave is -30.8 . Total momentum of the three scattered waves becomes $-19.75-5.77-3.79=-29.3$. The discrepancy between momenta before and after scattering is due to the presence of the breather seen in Fig. 5. The corresponding normalized value of the Hamiltonian of the incident wave is 166.3 , while the Hamiltonians of the waves after scattering amount to $153.9+8.9+3.2=166.0$.

In Fig. 6 the cross section of the right half of the rod is further reduced such that $S_{2} / S_{1}=0.3$, while $\alpha=1$ and $c=2$. In this case the incident solitary wave momentum -30.8 , and energy 166.3 are fissioned into three transmitted solitary waves, a reflected antisolitary wave, and a breather. In this case the total momentum of the scattered solitary waves becomes -30.3 , while the total energy is 165.4.

\section{DISCONTINUOUSLY VARYING CROSS SECTION AT MIBE}

In the case of discontinuously varying cross section, Eq. (9) yields the results shown in Fig. 7 for $S_{2} / S_{1}=0.49$ for an incident solitary wave (17) with $c=1.5$. As a result of the interaction with the discontinuity, two transmitted solitary waves, one reflected antisolitary wave, and a weak breather are produced. The momentum and energy of the incident wave are -17.3 and 45.3 , respectively, while the total momentum and energy of the three solitary waves become -20.7 and 45.1, respectively.

In Fig. 8 the same incident solitary wave hits a discontinuity $S_{2} / S_{1}=0.25$ giving rise to three transmitted solitary waves, one reflected antisolitary wave, and a somewhat stronger breather. In this case the momentum and energy of the incident wave are unchanged, while the corresponding quantities for the scattered solitary waves become -18.3 and 44.0 , respectively.

\section{ENDING ROD DESCRIBED BY MIBE}

For the semi-infinite rod, Eq. (10) yields Fig. 9 in the case of weak loading at the end $(m=5)$ corresponding to an almost free end, $w_{x}\left(x_{0}, t\right)=0$, implying $w_{x t t}\left(x_{0}, t\right)=0$. Again, the incident solitary wave is given by (17) with $c=1.5$. As a result, a reflected antisolitary wave plus a reflected breather are produced, the former traveling in the negative $x$ direction with velocity $c=1.44$.

Figure 10 shows the results for heavy loading at the end ( $m=25$ ) corresponding to an almost fixed end $\left[w\left(x_{0}, t\right)=0\right.$ implying $\left.w_{t t}\left(x_{0}, t\right)=0\right]$. In this case the incident solitary wave is reflected into a solitary wave followed by a sequence of antisolitary waves.

\section{CONCLUSION}

For IBE we have found blowup and dispersive destruction of negative solitary waves. In the MIBE we have shown that solitary waves can be transmitted, reflected, and fissioned at continuously and discontinuously varying cross sections as well as ends of semi-infinite rods. In the same cases traveling breathers are found. Conservation of momentum and energy is checked.

\footnotetext{
'M. P. Soerensen, P. L. Christiansen, and P. S. Lomdahl, “Solitary waves on nonlinear elastic rods. I," J. Acoust. Soc. Am. 76, 871-879 (1984).

${ }^{2} R$. S. Johnson, “On the development of a solitary wave moving over an uneven bottom," Proc. Cambridge Philos. Soc. 73, 183-203 (1973).

${ }^{3}$ V. D. Djordjevic and L. G. Redekopp, "The fission and disintegration of internal solitary waves moving over two-dimensional topography," J. Phys. Oceanogr. 8, 1016-1024 (1978).

${ }^{4} \mathrm{~K}$. Ko and H. H. Kuehl, "Energy loss of a Korteweg-de Vries solitary wave in a slowly varying medium," Phys. Fluids 23, 834-836 (1980).

${ }^{5}$ C. J. Knickerbocher and A. C. Newell, "Shelves and the Korteweg-de Vries equation," J. Fluid Mech. 98, 803-818 (1980).

"X-C. Zhong and M. C. Shen, "Fission of solutions in a symmetric triangular channel with variable cross section," Wave Motion 5, 167-176 (1983).

7. A. Molotkov and S. A. Vakulenko, "Nonlinear longitudinal waves in inhomogeneous rods" (in Russian), Zap. Nauchn. Semin. LOMI 99, 64 73 (1980).

${ }^{8}$ M. Peyrard, S. Pnevmatikos, and N. Flytzanis, "Discreteness effects on non-topological kink soliton dynamics in nonlinear lattices," preprint (1985).

'S. Pnevmatikos, N. Flytzanis, and M. Remoissenet, "Soliton dynamics of nonlinear diatomic lattices," preprint (1985).

${ }^{10}$ Y. Nejoh, K. Konno, and Y. H. Ichikawa, “A cusp solitary wave propagating in a nonlinear transmission line," Theor. Appl. Mech. 32, 533-538 (1984).

"H. A. Levine and B. D. Sleeman, "A note on the nonexistence of global solutions of initial-boundary value problems for the Boussinesq equation $u_{u}=3 u_{x x x x}+u_{x x}-12\left(u^{2}\right)_{x x}$," J. Math. Anal. Appl. 107, 206-210 (1985).

12J. L. Bona, W. G. Pritchard, and L. R. Scott, "A comparison of solutions of two model equations for long waves," Lect. Appl. Math. 20, 229-260 (1982).

${ }^{13} \mathrm{H}$. Sagan, Introduction to the Calculus of Variations (McGraw-Hill, New York, 1969).
} 